\title{
EVALUACIÓN DEL CONSUMO DE SEMILLAS DE MALEZAS POR NOTIOBIA (ANISOTARSUS) CUPRIPENNIS (GERM.) (COLEOPTERA: CARABIDAE) ${ }^{1}$
}

\author{
MARCELALIETTI' ${ }^{2}$, GUILLERMO MONTERO 2 , DELMAFACCINI ${ }^{3}$ y LUISANISENSOHN ${ }^{3}$
}

\begin{abstract}
RESUMEN - En este trabajo se estimó la preferencia alimentaria de Notiobia cupripennis por semillas de ocho especies de malezas primavero-estivales (Pr-Es) y de seis especies otoño-invernales (Ot-In) más comunes en los agroecosistemas del Sur de la provincia de Santa Fe, Argentina. Las semillas de las especies de cada grupo fueron ofrecidas en conjunto y de a pares a adultos confinados individualmente. La relación (semillas dañadas/semillas disponibles) x 100 de cada especie fue considerada como índice de preferencia alimentaria. Los resultados fueron analizados mediante análisis de la variancia y test de rangos múltiples de Duncan y mediante un test de diferencia de medias, previa transformación arcoseno de los datos. Las semillas de las especies Ot-In fueron más dañadas $(27,65 \%)$ que las Pr-Es $(10,40 \%)$. Se encontraron diferencias altamente significativas en el porcentaje de semillas dañadas entre las diferentes especies; las dicotiledóneas fueron más preferidas que las gramíneas. El orden de preferencia alimentaria se mantuvo cuando los insectos fueron obligados a elegir entre semillas de dos especies de malezas. La preferencia alimentaria se atribuiría a diferencias en el tamaño, la consistencia del tegumento y la composición química de las semillas en relación con la habilidad de manipulación de las mismas y los requerimientos nutricionales del insecto.
\end{abstract}

Términos para índice: predación de semillas, preferencia alimentaria, carábidos, agroecosistemas, Amaranthus quitensis.

\author{
CONSUMPTION EVALUATION OF WEED SEEDS \\ BY NOTIOBIA (ANISOTARSUS) CUPRIPENNIS (GERM.) (COLEOPTERA: CARABIDAE)
}

\begin{abstract}
The food preference of Notiobia cupripennis among seeds of eight weedy spring-summer species $(\mathrm{Sp}-\mathrm{Su})$ and six autumn-winter $(\mathrm{Av}-\mathrm{Wi})$ species more common in the agroecosystems of the South of Santa Fe Province, Argentina, was estimated in this study. The seeds of the species of each group were offered simultaneously and in pairs to adults individually placed. The relation (damaged seeds/available seeds) x 100 of each species was considered as a food preference index. The data were analysed by analysis of variance and Duncan's Multiple Range Test and by a paired difference test, previous arcsine-square-root transformation. The seeds of the Av-Wi species were more damaged (27.65\%) than the Sp-Su ones (10.40\%). Highly significative differences in the percentage of damaged seeds among the different species were found; dicotyledones were preferred over gramines. The food preference ranking was maintained when the insects were forced to choose between the seeds of two weed species. The food preference would be attributed to differences in seed size, tegument toughness and chemical composition of the seeds in relation to the ability of handling the seeds and the nutritional requirements of the insect.
\end{abstract}

Index terms: seed predation, food preference, carabids, agroecosystems, Amaranthus quitensis.

\footnotetext{
${ }^{1}$ Aceptado para publicación en 11 de junio de 1999.

${ }^{2}$ Ing. Agr., Cátedra de Zoología Agrícola, Facultad de Ciencias Agrarias, U.N.R., C.C. 14. 2123. Zavalla, Santa Fe, Argentina.E-mail: mlietti@ffcagr.unr.edu.ar

${ }^{3}$ Ing. Agr., Cátedra de Malezas, Facultad de Ciencias Agrarias, U.N.R.
}

\section{INTRODUCCIÓN}

Los bancos de semillas de malezas se ven afectados por pérdidas producidas por germinación, muerte fisiológica de las semillas provocada por la senescencia natural, y predación (Harper, 1977; 
Simpson et al., 1989). La predación tiene un rol importante en la reducción del número de semillas del banco de algunas especies de malezas (Scopel et al., 1988; Van Esso \& Ghersa, 1989; Dellafiore \& Polov, 1994).

Dentro de la fauna de predadores que habitan en el suelo en agroecosistemas, los carábidos son importantes agentes de control natural al predar sobre plagas insectiles (Best \& Beegle, 1977a; Barney \& Pass, 1986) y al alimentarse de plantas no deseadas, particularmente semillas de malezas (Best \& Beegle, 1977b; Lund \& Turpin, 1977; Barney \& Pass, 1986; Brust \& House, 1988; Cardina et al., 1996). Se conocen numerosos carábidos que consumen material vegetal en diferentes proporciones (Johnson \& Cameron, 1969; Hengeveld, 1980).

Los adultos de las especies sudamericanas de Anisotarsus son aparentemente oportunistas o fitófagos en sus hábitos alimentarios (Noonan, 1981). En la República Argentina, adultos de $N$. cupripennis han sido observados por Bosq (1942) alimentándose de granos recién maduros de cebada y centeno y por Ahmad (1974) atacando a la larva del gorgojo de la alfalfa Graphognatus leucoloma (Boh.). Nisensohn et al. (1999) evaluaron indirectamente la predación en el campo de semillas de Amaranthus quitensis L. (H.B.K.) producida por este insecto. De lo anteriormente mencionado surge que se conoce muy poco acerca de la composición de la dieta de esta especie y en consecuencia poco se puede inferir sobre su rol en el agroecosistema.

$N$. cupripennis es una especie de hábito crepuscular (Bosq, 1942) o nocturno (Noonan, 1981) y en consecuencia realizar una observación directa a campo sobre su comportamiento alimentario de elementos de pequeño tamaño como son las semillas de malezas resultaría experimentalmente difícil de resolver y costoso en término de las horas-hombre requeridas. Luego un estudio realizado en el laboratorio es muy útil para la obtención de información sobre la capacidad de consumo y las preferencias alimentarias de esta especie, la cual es necesaria antes de evaluar su impacto como agente de control natural de propágulos de malezas específicas.
El objetivo de este estudio es estimar la preferencia alimentaria de $N$. cupripennis por semillas de las especies de malezas primavero-estivales (Pr-Es) y otoño-invernales (Ot-In) más comunes en los agroecosistemas del sur de la provincia de Santa Fe, Argentina.

\section{MATERIAL Y MÉTODOS}

Los insectos adultos y las semillas utilizadas en los experimentos fueron colectados del campo durante la semana previa al inicio de los mismos. Los insectos fueron mantenidos en el laboratorio hasta el día previo a su utilización en bandejas de plástico de $35 \times 25 \times 5 \mathrm{~cm}$, provistas de un papel secante humedecido en su base, con una mezcla de semillas de las especies de malezas suministradas en los experimentos.

\section{Preferencia alimentaria entre especies de malezas primavero-estivales u otoño-invernales}

Se realizaron dos experimentos para determinar la preferencia alimentaria por semillas de ocho especies de malezas de ciclo Pr-Es o de seis especies de malezas de ciclo Ot-In.

Las especies Pr-Es seleccionadas fueron: yuyo colorado (Amaranthus quitensis L. (H.B.K.)), malva cimarrona (Anoda cristata (L.)), amor seco (Bidens subalternans D.C.), quinoa (Chenopodium album L.), chamico (Datura ferox $\mathrm{L}$.), pasto cuaresma (Digitaria sanguinalis (L.)), capín (Echinochloa colonum (L.) Link.), cola de zorro (Setaria geniculata (Lam.)). Las especies Ot-In utilizadas fueron: bowlesia (Bowlesia incana Ruiz et Pav.), bolsa del pastor (Capsella bursa-pastoris (L.)), mastuerzo (Coronopus didymus (L.)), ray-grass criollo (Lollium multiflorum Lam.), capiquí (Stellaria media (L.)), lengua de vaca (Rumex crispus L.).

En cada uno de los experimentos, ocho insectos adultos (cuatro hembras y cuatro machos) fueron confinados individualmente en una bandeja plástica de $35 \times 25 \times 5 \mathrm{~cm}$ cubierta con una bolsa de celofán. En la base del recipiente se colocó un papel secante humedecido y sobre este se distribuyeron al azar 20 semillas agrupadas de cada especie de maleza. Se proveyó de hojas secas como lugar de refugio en el centro del recipiente. Al cabo de 48 horas se examinaron las semillas bajo lupa estereoscópica con 10 aumentos y se registró el número de semillas no dañadas de cada especie. Fueron consideradas dañadas las semillas con el tegumento partido, los cotiledones total o parcialmente comidos y con las glumas abiertas con el grano total o 
parcialmente comido en su interior. El daño ocasionado a las semillas en los experimentos de laboratorio fue considerado como un criterio de preferencia alimentaria.

Se calculó para cada especie un índice de preferencia alimentaria en base a la relación (semillas dañadas/semillas disponibles presentes inicialmente) $x$ 100. Los resultados fueron analizados mediante un análisis de la varianza para un diseño jerárquico-factorial (los individuos jerarquizados dentro del sexo y como factores el sexo y la especie de semilla) y test de rangos múltiples de Duncan, previa transformación arcoseno de los datos, a través del procedimiento General Lineal Models (G.L.M.) (SAS Institute, 1982).

\section{Preferencia alimentaria entre dos especies}

Se determinó la preferencia alimentaria cuando la disponibilidad de alimento en el ambiente se restringía a las semillas de dos especies de las malezas más dañadas en los experimentos previos.

Las semillas de cuatro especies de malezas Ot-In (C. bursa-pastoris, C. didymus, S. media, L. multiflorum) y de cinco especies de malezas Pr-Es (A. quitensis, $D$. sanguinalis, $C$. album, E. colonum, $S$. geniculata) fueron seleccionadas para realizar un total de seis y cuatro comparaciones de a pares respectivamente. En los sistemas de labranza cero las semillas de las especies de malezas Pr-Es permanecen sobre la superficie del suelo junto con las semillas de las especies Ot-In; por este motivo la especie de maleza Pr-Es más preferida fue seleccionada para realizar comparaciones de a pares con las semillas de las tres malezas Ot-In más dañadas (C. bursa-pastoris, C. didymus, S. media).

Diez insectos adultos (cinco hembras y cinco machos) fueron confinados individualmente en recipientes de plástico transparente con tapa, de $8,5 \times 8,5 \times 5 \mathrm{~cm}$; en el fondo de los cuales se colocaron 20 semillas de cada una de las dos especies de malezas a comparar sobre un papel secante humedecido. Después de 48 horas, se examinaron las semillas bajo lupa estereoscópica con 10 aumentos y se registró el número de semillas no dañadas. Cuando se comparó el consumo de semillas de A. quitensis y C. album se colocaron 50 semillas de cada una durante un período de 24 horas para que queden semillas sin consumir y se manifiesten las posibles diferencias en preferencia. Se estimó un índice de preferencia alimentaria para cada especie de acuerdo a la fórmula utilizada en los dos experimentos previos. La diferencia del porcentaje de semillas de malezas dañadas de cada par de especies fue comparada utilizando un test de diferencia de medias, previa transformación arcoseno de los datos, a través del procedimiento MEANS (SAS Institute, 1982).
La distancia máxima de apertura de las mandíbulas de cinco hembras y de cinco machos utilizados en los experimentos se midió por medio de un calibre. Los resultados fueron expresados como valor promedio \pm D.S. El tamaño de las semillas de las especies seleccionadas fue extraído de Petetín \& Molinari (1982).

Los recipientes conteniendo los insectos y semillas en todos los experimentos previos fueron colocados en una cámara climatizada a una temperatura de $25 \pm 2{ }^{\circ} \mathrm{C}$ y fotoperíodo de 11,5 horas.

\section{Consumo de semillas de $A$. quitensis}

Se determinó la tasa de consumo diaria de semillas de $A$. quitensis a 24 y $22^{\circ} \mathrm{C}$. Estas temperaturas son levemente superiores a las temperaturas medias mensuales del suelo a $5 \mathrm{~cm}$ de profundidad del mes de marzo $\left(23^{\circ} \mathrm{C}\right)$ y abril $\left(19^{\circ} \mathrm{C}\right)$ respectivamente, que corresponde con el período de mayor predación a campo de esta especie.

En el primer caso tuvo una duración de cinco días y se utilizaron cuatro machos y cuatro hembras, mientras que el segundo se prolongó por ocho días y se emplearon seis machos y seis hembras. Cada insecto adulto fue confinado en un recipiente de plástico transparente con tapa, de $8,5 \times 8,5 \times 5 \mathrm{~cm}$; en el fondo del cual se colocaron diariamente 100 semillas de $A$. quitensis sobre un papel secante humedecido. Después de 24 horas, se examinaron las semillas bajo lupa estereoscópica con 10 aumentos y se registró el número de semillas no dañadas. El consumo de semillas (número de semillas total o parcialmente comidas) se obtuvo por diferencia entre la cantidad de semillas colocadas y la de semillas totalmente sanas encontradas al cabo de 24 horas. Los recipientes con los insectos fueron colocados en una cámara climatizada a las temperaturas indicadas y con un fotoperíodo de 11,5 horas. Los resultados fueron analizados mediante un análisis de la varianza para un diseño en parcela dividida, siendo la parcela principal el día de consumo y la subparcela el sexo, y test de rangos múltiples de Duncan, previa transformación arcoseno de los datos, a través del procedimiento General Lineal Models (G.L.M.) (SAS Institute, 1982). Los mismos fueron expresados como el valor promedio \pm E.S.

\section{RESULTADOS Y DISCUSIÓN}

\section{Preferencia alimentaria entre especies de malezas} primavero-estivales u otoño-invernales

Las semillas de las especies de malezas de ciclo Ot-In fueron más dañadas $(27,65 \%)$ que las de ciclo Pr-Es $(10,40 \%)$. Se encontraron diferencias 
altamente significativas en el porcentaje de semillas dañadas entre las diferentes especies de malezas Ot-In $(\mathrm{F}=4,76 ; \mathrm{gl}=5,30 ; \mathrm{p}<0,01)$ y Pr-Es $(\mathrm{F}=8,20$; $\mathrm{gl}=7,42 ; \mathrm{p}<0,01)$, siendo las diferencias en preferencia más notables entre las especies de este ciclo biológico. No hubo diferencias significativas en el consumo de semillas entre hembras y machos. La interacción entre tipos de semillas y sexo no fue significativa para ambos grupos de especies de malezas.

$N$. cupripennis consumió las semillas de cinco de las ocho especies de malezas de ciclo Pr-Es ofrecidas, aunque las de $A$. quitensis (39,75\%), C. album $(23,13 \%)$ y $D$. sanguinalis $(15,30 \%)$ fueron más dañadas que las del resto de las especies, cuyo porcentaje promedio de semillas dañadas no fue estadísticamente diferente de cero $(\mathrm{p}>0,19)$. Las semillas de $A$. quitensis fueron significativamente más preferidas que las de C. album y D. sanguinalis, siendo estas últimas igualmente seleccionadas. Las semillas de $B$. subalternans, $D$. ferox y $A$. cristata no fueron dañadas (Cuadro 1).

$N$. cupripennis consumió en mayor grado las semillas de cuatro de las seis especies de malezas de ciclo Ot-In ofrecidas, las cuales en orden decreciente de preferencia fueron: C. bursa-pastoris $(49,55 \%)$, C. didymus $(45,55 \%)$, S. media (34,39\%), L. multiflorum $(20,48 \%)$. El porcentaje promedio de semillas dañadas de $B$. incana y $R$. crispus no fue estadísticamente diferente de cero $(\mathrm{p}>0,09)$. Las semillas de $C$. bursa-pastoris y $C$. didymus fueron significativamente más consumidas que las de $B$. incana y $R$. crispus, siendo igualmente preferidas que las de las restantes especies (Cuadro 1).

Johnson \& Cameron (1969) reportaron una lista de 150 especies de carábidos conocidos por alimentarse de materia vegetal en diferentes proporciones. De esta lista surge que el hábito alimentario fitófago está concentrado en los miembros de la tribu Harpalini, y en algunos géneros de otras tribus (Amara y Pterostichus). Cuando se alimentan de semillas son capaces de consumir selectivamente semillas de determinadas especies de malezas (Lund \& Turpin, 1977; Brust \& House, 1988; Brust, 1994). En Estados Unidos, Harpalus pensylvanicus De Geer, un harpalini frecuente en cultivos de maíz, soja y alfalfa, es omnívoro en sus hábitos alimentarios, consumiendo tanto semillas de malezas como insectos muertos y vivos (Best \& Beegle, 1977a; Best \& Beegle, 1977b; Lund \& Turpin, 1977; Barney \& Pass, 1986). El único registro que se tiene de $N$. cupripennis como predador de insectos es atacando a la larva del gorgojo de la alfalfa G. leucoloma (Ahmad, 1974). En este estudio se amplia el rango de especies vegetales que es capaz de aceptar y consumir este insecto, el cual estaba limitado a granos recién maduros de cebada y centeno (Bosq, 1942).

La utilización de semillas como principal fuente de alimentación es un hábito típico de ambientes desérticos, semidesérticos y mediterráneos por las hormigas cosechadoras de la subfamilia Myrmicinae (Sudd \& Franks, 1987; Louda, 1995). Varios investigadores han sugerido que el consumo selectivo de

CUADRO 1. Consumo de semillas de ocho especies de malezas de ciclo primavero-estival y de seis species de malezas de ciclo otoño-invernal por $N$. cupripennis. Promedio de ocho individuos.

\begin{tabular}{lcc}
\hline Maleza & $\begin{array}{c}\text { Composición } \\
\text { dieta }\end{array}$ & $\begin{array}{c}\text { Semillas } \\
\text { dañadas }\end{array}$ \\
\hline Primavero-estivales & \\
A. quitensis & 49,92 & $39,75 \mathrm{a}$ \\
C. album & 26,60 & $23,13 \mathrm{~b}$ \\
D. sanguinalis & 17,50 & $15,30 \mathrm{bc}$ \\
E. colonum & 4,76 & $2,50 \mathrm{~cd}$ \\
S. geniculata & 1,22 & $1,94 \mathrm{~d}$ \\
B. subalternans & 0,00 & $0,00 \mathrm{~d}$ \\
D. ferox & 0,00 & $0,00 \mathrm{~d}$ \\
A. cristata & 0,00 & $0,00 \mathrm{~d}$ \\
Total & 100,00 & 10,40 \\
\hline & Otoño-invernales & \\
C. bursa-pastoris & 35,88 & $49,55 \mathrm{a}$ \\
C. didymus & 24,35 & $45,55 \mathrm{a}$ \\
S. media & 21,08 & $34,39 \mathrm{ab}$ \\
L. multiflorum & 11,78 & $20,48 \mathrm{abc}$ \\
B. incana & 6,14 & $13,75 \mathrm{bc}$ \\
R. crispus & 0,77 & $1,88 \mathrm{c}$ \\
Total & 100,00 & $27,65 \%$ \\
\hline Los porcen & . & \\
\hline
\end{tabular}

${ }^{1}$ Los porcentajes no seguidos por la misma letra son significativamente diferentes al nivel del 5\%. Test de rangos múltiples de Duncan. 
semillas está determinado por numerosos factores como la abundancia en el ambiente durante la época de recolección (Tevis, 1958; Whitford, 1978), el tamaño y la disposición espacial (Brown et al., 1975; Whitford, 1978), las características físico-mecánicas relacionadas con la consistencia y textura del tegumento (Brown et al., 1975), la composición química (Whitford, 1978; Gordon, 1980; Sudd \& Franks, 1987) y la calidad nutricional (Whitford, 1978).

En los experimentos de comparación entre semillas de varias especies de malezas de ciclo Pr-Es y Ot-In los grupos de semillas de cada especie fueron distribuidos al azar. Si bien la disposición espacial de las semillas fue agregada, consideramos que la distancia entre grupos de semillas ( 7 a $9 \mathrm{~cm})$ es pequeña en relación con la capacidad de movimiento de este insecto, con lo cual estimamos que los insectos tuvieron la oportunidad de visitar todos los grupos de semillas.

El tamaño y la forma de las semillas junto con la dureza y textura del tegumento tienen efecto sobre la habilidad del insecto para atrapar, manipular y morder con sus mandíbulas las semillas de las diferentes especies. En agroecosistemas sojeros, los carábidos más pequeños $(<15 \mathrm{~mm})$ consumieron casi exclusivamente las semillas de malezas de menor tamaño (Brust \& House, 1988). En nuestro experimento las semillas de mayor tamaño y dureza ( $B$. subalternans, $D$. ferox y $A$. cristata) no fueron consumidas. Sus dimensiones exceden el tamaño de apertura máxima de las mandíbulas de las hembras $(1,69 \mathrm{~mm} \pm 0,05)$ y de los machos $(1,70 \mathrm{~mm} \pm 0,07)$ (Cuadro 2). En cambio, este insecto parte fácilmente con las mandíbulas las semillas de las especies Pr-Es y Ot-In más preferidas. Las semillas más pequeñas como A. quitensis y C. album son comidas totalmente, quedando fragmentos pequeños de estas semillas esparcidos por el recipiente; los restos del tegumento no son digeridos encontrándose en los excrementos. En el caso de las semillas de $D$. sanguinalis el insecto abre las glumas y consume el grano; mientras que los granos de las otras gramíneas Pr-Es (S. geniculata y E. colonum) y Ot-In (L. multiflorum) menos preferidas son consumidos a través de las glumas, sin abrirlas. Nuevamente la diferencia en el comportamiento de consumo podría deberse a la dureza y tenacidad de las glumas de estas tres especies con respecto a la primera. De la semilla de $B$. incana, una especie Ot-In poco preferida, come los cotiledones dejando el tegumento.

Generalmente las semillas son más nutritivas que las partes verdes de la planta por tener cantidades relativamente más altas de proteína. Sin embargo, frecuentemente tienen un alto contenido de metabolitos secundarios que afectan la aceptabilidad por parte de los insectos (Janzen, 1971; Bernays \& Chapman, 1994). La localización y el consumo de semillas por los predadores de semillas involucra la presencia de sustancias químicas atractantes, repelentes, disuasivas, tóxicas y fagoestimulantes (Janzen, 1971). El género Datura contiene alcaloides, Gossypium (Malvaceae) y Poligonum (Poligonaceae) terpenoides, las Compositae alcaloides y terpenoides y las Umbelliferae furanocumarinas (Bernays \& Chapman, 1994). Gordon (1980) sugirió que el contenido de lípidos superficiales junto con monoterpenoides en el tegumento, el cual estaba altamente correlacionado con el contenido calórico interno, sería el estímulo químico que podría ser utilizado por las hormigas en

CUADRO 2. Tamaño de las semillas de especies de malezas de ciclo primavero-estival y otoño-invernal más comunes en los agroecosistemas del Sur de la Provincia de Santa Fe, Argentina (Petetin \& Molinari, 1982).

\begin{tabular}{llc}
\hline Espécie & \multicolumn{1}{c}{ Família } & Tamaño (mm) \\
\hline & & Primavero-estival \\
A. quitensis & Amaranthaceae & $1 \times 0,7$ \\
D. sanguinalis & Gramineae & $3,20 \times 0,64$ \\
C. album & Chenopodiaceae & $1 \times 0,7$ \\
E. colonum & Gramineae & $3,14 \times 1,60$ \\
S. geniculata & Gramineae & $2,2-2,8($ largo $)$ \\
B. subalternans & Compositae & $10,84 \times 0,64$ \\
D. ferox & Solanacea & $4,58 \times 2,12$ \\
A. cristata & Malvacea & $3-5$ \\
\hline & & Otoño-invernal \\
C. bursa-pastoris & Cruciferae & $0,7-1$ \\
C. didymus & Cruciferae & $1-1,5$ \\
S. media & Cariophyllaceae & $1 \times 0,7$ \\
L. multiflorum & Gramineae & $5-5,5 \times 1-1,2$ \\
B. incana & Umbelliferae & $1,5-2,5 \times 1-2$ \\
R. crispus & Polygonaceae & $4-4,5$ \\
\hline & &
\end{tabular}


la selección de las semillas. Las Cruciferae contienen glucocinolatos, las cuales son sustancias químicas repelentes para insectos que no se alimentan de las mismas y fagoestimulantes para insectos que prefieren estas plantas (Bernays \& Chapman, 1994). Las dos semillas más preferidas dentro del grupo de especies Ot-In pertenecen a la familia Cruciferae. La preferencia alimentaria podría atribuirse a diferencias en el tamaño y consistencia del tegumento, junto con la presencia de compuestos químicos secundarios. De este modo el insecto muerde el perigonio de los frutos de $R$. crispus $\sin$ llegar a afectar la semilla que se encuentra en su interior.

$N$. cupripennis prefirió significativamente las semillas de especies de malezas dicotiledóneas con respecto a las de gramíneas. Brust (1994) demostró que los predadores de semillas (grillos y carábidos), al consumir preferentemente o eliminar proporcionalmente más semillas de malezas de hoja ancha (A. retroflexus L. y C. album) con respecto a las de gramíneas (D. sanguinalis y Panicum dichotolorum L.), disminuyeron la biomasa individual relativa y en consecuencia redujeron la habilidad competitiva de las latifoliadas cuando crecían con las gramíneas. Otros predadores de semillas como las hormigas al consumir selectivamente ciertas especies de gramíneas disminuyeron la biomasa total de las malezas y produjeron un cambio en el resultado de la competencia interespecífica (Risch \& Carroll, 1986).

\section{Preferencia alimentaria entre dos especies}

El orden de preferencia al comparar pares de especies de malezas fue igual al observado en las comparaciones entre varias especies juntas. Sin embargo, en general, se pusieron de manifiesto mayores diferencias en preferencia entre las especies. Las semillas de $C$. album fueron significativamente más preferidas que las de $D$. sanguinalis $(\mathrm{p}=0,02)$ y las de $S$. geniculata con respecto a las de E. colonum $(\mathrm{p}<0,01)$. Las semillas de $A$. quitensis fueron igualmente preferidas que las de C. album (Cuadro 3). Dentro del grupo de especies de malezas de ciclo Ot-In, las semillas de $S$. media fueron significativamente más preferidas que las de L. multiflorum ( $\mathrm{p}<0,01)$, y las semillas de C. bursa-pastoris fueron significativamente más dañadas con respecto a las de $S$. media y L. multiflorum $(\mathrm{p}<0,01)$ (Cuadro 3). Las semillas de A. quitensis fueron significativamente más dañadas que el resto de las semillas de malezas de ciclo Ot-In $(\mathrm{p}<0,01)$ (Cuadro 3).

El grado de preferencia por las semillas de una especie puede estar afectado por el tipo y la cantidad de semillas de otras especies en el mismo ambiente (Risch \& Carroll, 1986). Dentro del grupo de especies de malezas de ciclo Pr-Es, las semillas de especies menos preferidas, como $C$. album, $D$. sanguinalis y $S$. geniculata, fueron proporcionalmente más dañadas que las semillas de la especie más preferida (A. quitensis) cuando disminuye la disponibilidad total de semillas en el ambiente. No ocurre lo mismo dentro del grupo de especies Ot-In, cuyo porcentaje de semillas dañadas no varía o lo hace levemente cuando cambia su proporción en el ambiente. Sin embargo, las semillas de $A$. quitensis fueron proporcionalmente más consumidas cuando se encuentran con las semillas de especies Ot-In. Por otro lado cuando D. sanguinalis está presente los insectos seleccionan proporcionalmente más semillas de C. album que de A. quitensis. Luego dependiendo de la combinación de especies de malezas presentes en un área, la predación diferencial por parte de estos insectos puede provocar que ciertas malezas se establezcan mejor que otras en un microhabitat en particular (Brust, 1994).

\section{Consumo de semillas de $A$. quitensis}

El consumo diario de semillas de $A$. quitensis fue de $61,50 \pm 7,19$ (hembras) y 27,10 $\pm 4,62$ (machos) a $24^{\circ} \mathrm{C}$; y de $38,27 \pm 4,01$ (hembras) y $23,96 \pm 2,24$ (machos) a $22^{\circ} \mathrm{C}$ de temperatura. Luego, las hembras consumieron 2,3 veces y 1,6 veces más semillas que los machos a 24 y $22^{\circ} \mathrm{C}$ respectivamente. Las diferencias en el consumo de semillas entre hembras y machos fueron altamente significativas a $24^{\circ} \mathrm{C} \quad(\mathrm{F}=12,78 ; \mathrm{gl}=1,15$; $\mathrm{p}<0,01)$ y significativas a $22^{\circ} \mathrm{C} \quad(\mathrm{F}=6,25$; $\mathrm{gl}=1,40 ; \mathrm{p}=0,02)$. El consumo diario de semillas disminuyó en el tiempo; obteniéndose diferencias significativas entre los días de consumo tanto a la 
CUADRO 3. Preferencia alimentaria de $N$. cupripennis por semillas de especies de malezas de ciclo primavero-estival y otoño-invernal ofrecidas de a pares. Promedio de 10 individuos.

\begin{tabular}{|c|c|c|c|}
\hline \multirow[t]{2}{*}{ Pares de especies ${ }^{1}$} & \multicolumn{3}{|c|}{ Porcentaje de semillas dañadas } \\
\hline & $1^{\circ} \mathrm{sp}$. & $2^{-0} \mathrm{sp}$. & Diferencia \\
\hline \multicolumn{4}{|c|}{ Malezas primavero-estivales } \\
\hline A. quitensis x C. album & 70,40 & 66,20 & $4,20^{\mathrm{ns}}$ \\
\hline A. quitensis $\times$ D. sanguinalis & 58,50 & 35,00 & $23,50 *$ \\
\hline C. album $\times$ D. sanguinalis & 65,50 & 32,48 & $33,02 *$ \\
\hline S. geniculata $\times$ E. colonum & 76,00 & 00,00 & $76,00 * *$ \\
\hline \multicolumn{4}{|c|}{ Malezas otoño-invernales } \\
\hline C. bursa-pastoris x $S$. media & 66,76 & 34,29 & $32,46^{* *}$ \\
\hline C. didymus $\mathrm{x}$ C. bursa-pastoris & 56,00 & 55,68 & $0,32^{\mathrm{ns}}$ \\
\hline C. didymus x $S$. media & 54,50 & 31,37 & $23,13^{\mathrm{ns}}$ \\
\hline C. bursa-pastoris $\mathrm{x}$ L. multiflorum & 58,00 & 4,50 & $53,50 * *$ \\
\hline S. media $\times$ L. multiflorum & 27,06 & 5,50 & $21,56^{* *}$ \\
\hline C. didymus $\mathrm{x}$ L. multiflorum & 20,75 & 7,93 & $12,82^{\mathrm{ns}}$ \\
\hline \multicolumn{4}{|c|}{ A. quitensis-Malezas otoño-invernales } \\
\hline A. quitensis $\mathrm{x}$ C. bursa-pastoris & 100,00 & 48,50 & $51,50 * *$ \\
\hline A. quitensis $\times$ C. didymus & 99,00 & 42,50 & $56,50 * *$ \\
\hline A. quitensis $\mathrm{x}$ S. media & 100,00 & 26,50 & $73,50 * *$ \\
\hline
\end{tabular}

${ }^{1}$ La semilla preferida en este experimento está colocada en primer lugar.

$*^{* *} \mathrm{p}<0,01 ; * 0,01<\mathrm{p}<0,05$; $^{\text {ns }}$ no significativo, $\mathrm{p}>0,10$.

mayor $(\mathrm{F}=3,77 ; \mathrm{gl}=4,15 ; \mathrm{p}=0,02)$ como a la menor temperatura $(\mathrm{F}=3,02 ; \mathrm{gl}=7,40 ; \mathrm{p}=0,012)$. La cantidad de semillas comidas fue significativamente mayor en el primer día. Las hembras consumieron 1,6 veces y los machos 1,1 veces más semillas de $A$. quitensis a 24 que a $22^{\circ} \mathrm{C}$ (Figs. 1 y 2 ).

Las hembras requieren más nutrientes en el periodo de maduración sexual, lo cual puede conducir a cambios en el comportamiento alimentario y en la elección de la planta hospedadora, con respecto a los machos, para satisfacer sus requerimientos nutricionales (Bernays \& Chapman, 1994). Las hembras de $N$. cupripennis consumieron significativamente más semillas de $A$. quitensis que los machos. Estas hembras fueron colectadas del campo durante los meses de marzo y abril que coincide con el período reproductivo de esta especie. Además, $A$. quitensis es la especie más preferida tanto entre las especies de malezas Pr-Es como con respecto a las especies de malezas Ot-In. Las semillas de Amaranthus son comparables a las de los cereales en el contenido de carbohidratos, pero son superiores en el contenido de proteínas y lípidos, estos se encuentran en la forma de triglicéridos de ácidos grasos (Levin, 1974; Weaver \& McWilliams, 1980). La proteína constituye el principal nutriente requerido por los insectos fitófagos y se encuentra generalmente en cantidades limitantes para el crecimiento óptimo de los mismos (Bernays \& Chapman, 1994), mientras que los ácidos grasos constituyen la principal reserva de energía. Las semillas de $A$. retroflexus fueron también severamente dañadas por $H$. pensylvanicus cuando fueron ofrecidas individualmente, prefiriendo las semillas de Setaria sp. sobre otras especies en las comparaciones de a pares (Lund \& Turpin, 1977).

$N$. cupripennis consumió mayor cantidad de semillas de $A$. quitensis cuando se la ofrece como único alimento que cuando se la encuentra en el ambiente con otro tipo de semillas. Esto está relacionado con la disminución del consumo diario en el tiempo. La disminución en el consumo de una especie en el corto plazo es un patrón característico 


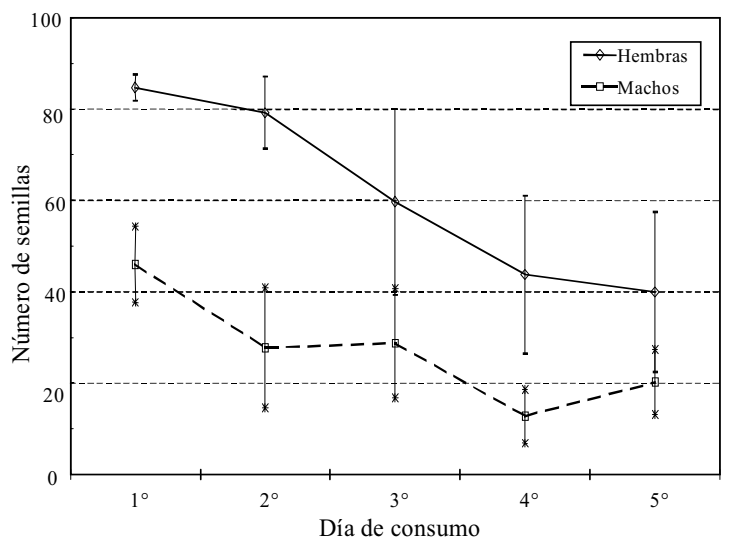

FIG. 1. Consumo diario promedio de semillas de $A$. quitensis por $N$. cupripennis durante cinco días. Temperatura: $24^{\circ} \mathrm{C}$. Las barras representan el error estandard de la media.

de insectos que seleccionan su hospedadora para obtener recursos complementarios (Howard, 1993; Bernays \& Chapman, 1994). El consumo de semillas de $A$. quitensis fue mayor a la temperatura correspondiente al mes de marzo que al mes de abril. Estos resultados coinciden con las tasas de predación obtenidas en el campo por Nisensohn et al. (1999), las cuales alcanzan los máximos valores entre el 15 y el 30 de marzo, para luego disminuir a partir del mes de abril.

\section{CONCLUSIONES}

1. N. cupripennis es capaz de consumir preferencialmente semillas de varias especies de malezas abundantes en agroecosistemas del sur de la provincia de Santa Fe.

2. Las semillas de las especies de malezas dicotiledóneas son preferidas a las de las gramíneas.

3. A. quitensis es la especie más preferida tanto entre las especies de malezas de ciclo primaveroestival como con respecto a las especies de malezas de ciclo otoño-invernal.

4. La proporción de semillas consumidas de A. quitensis depende de la presencia de semillas de otras especies en el mismo ambiente, siendo mayor cuando se la ofrece como único alimento.

5. Se amplia el rango de especies consumidas por este insecto.

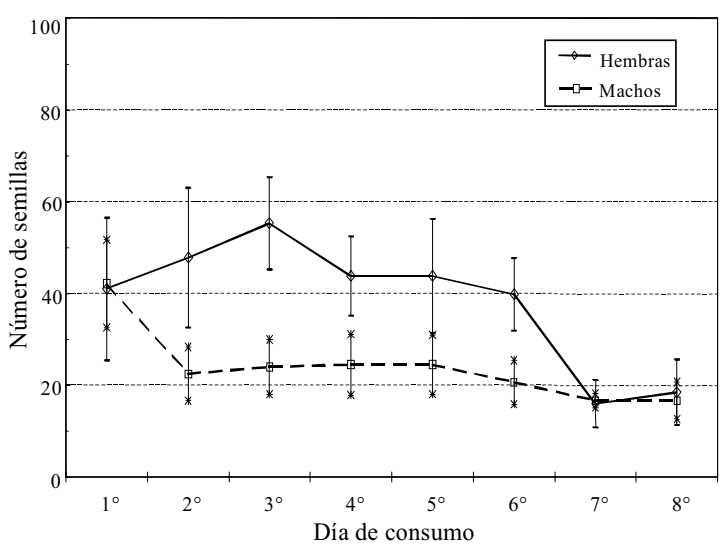

FIG. 2. Consumo diario promedio de semillas de $A$. quitensis por $N$. cupripennis durante ocho días. Temperatura: $22^{\circ} \mathrm{C}$. Las barras representan el error estandard de la media.

\section{AGRADECIMIENTOS}

A las estadísticas Graciela Giubileo y Vilma Bisaro de la Cátedra de Estadística de la Facultad de Ciencias Agrarias, U.N.R. por el asesoramiento brindado en el análisis estadístico de los resultados.

\section{REFERÊNCIAS}

AHMAD, R. Studies on Graphognathus leucoloma (Boh.) Col.:Curculionidae and its natural enemies in the central provinces of Argentina. Ottawa, Farham Royal : Commonwealth Agricultural Bureau, Commonwealth Institute of Biological Control, 1974. p.19-28. (Technical bulletin, n.17).

BARNEY, R.J.; PASS, B.C. Foraging behavior and feeding preference of ground beetles (Coleoptera: Carabidae) in Kentucky alfalfa. Journal of Economic Entomology, v.79, n.5, p.1334-1337, Oct. 1986.

BERNAYS, E.A.; CHAPMAN, R.F. Host-plant selection by phytophagous insects. New York: Chapman \& Hall, 1994. 312p.

BEST, R.L.; BEEGLE, C.C. Consumption of Agrotis ipsilon by several species of carabids found in Iowa. Environmental Entomology, v.6, n.4, p.532-534, Aug. 1977a.

BEST, R.L.; BEEGLE, C.C. Food preferences of five species of carabids commonly found in Iowa 
cornfields. Environmental Entomology, v.6, n.1, p.9-12, Feb. 1977 b.

BOSQ, J.M. Segunda lista de Coleópteros de la República Argentina dañinos a la agricultura. Ingeniería Agronómica, Buenos Aires, v.4, n.18, p.17-26, ene./feb. 1942.

BROWN, J.H.; GROVER, J.J.; DAVIDSON, D.W.; LIEBERMAN, G.A. A preliminary study of seed predation in desert and montane habitats. Ecology, v.56, n.4, p.987-992, summer 1975.

BRUST, G.E. Seed predators reduce broadleaf weed growth and competitive ability. Agriculture, Ecosystems and Environment, v.48, p.27-34, 1994.

BRUST, G.E.; HOUSE, G.J. Weed seed destruction by arthropods and rodents in low-input soybean agroecosystems. American Journal of Alternative Agriculture, v.3, n.1, p.19-25, 1988.

CARDINA, J.; NORQUAY, H.M.; STINNER, B.S.; McCARTNEY, D.A. Postdispersal predation of velvetleaf (Abutilon theophrasti) seeds. Weed Science, v.44, n.3, p.534-539, Jul./Sept. 1996.

DELLAFIORE, C.M.; POLOV, J. Feeding habitats of Calomys musculinus in the crop field and its borders. Mastozoología Neotropical, v.1, n.1, p.45-50, 1994.

GORDON, S.A. Analysis of twelve sonorant desert species preferred by the desert harvester ant. Madroño, v.27, n.2, p.68-78, Apr. 1980.

HARPER, J.L. Population biology of plants. London : Academic, 1977. 582p.

HENGEVELD, R. Qualitative and quantitative aspects of the food of ground beetles (Coleoptera: Carabidae): a review. Netherlands Journal of Zoology, v.30, n. 4, p.555-563, 1980.

HOWARD, J.H. Temporal pattern of resource use and variation in diets of individual grasshoppers (Orthoptera: Acrididae). Journal of Insect Behavior, v.6, n.4, p.441-453, 1993

JANZEN, D.H. Seed predation by animals. Annual Review of Ecological Systems, v.2, p.465-492, 1971.
JOHNSON, N.E.; CAMERON, R.S. Phytophagous ground beetles. Annals of the Entomological Society of America, v.62, n.4, p.909-914, July 1969.

LEVIN, D.A. The oil content of seeds: an ecological perspective. The American Naturalist, v.108, n.960, p.193-206, Mar./Apr. 1974.

LOUDA, S.M. Effect of seed predation on plant regeneration: evidence from pacific basin Mediterranean scrub communities. In: ARROYO, M.T.K.; ZEDLER, P.H.; FOX, M.D. (Eds.). Ecology and Biogeography of Mediterranean Ecosystems in Chile, California and Australia. New York : Springer-Verlag, 1995. Ch.13, p.311-344.

LUND, R.D.; TURPIN, F.T. Carabid damage to weed seeds found in Indiana cornfields. Environmental Entomology, v.6, n.5, p.695-698, Oct. 1977.

NISENSOHN, L.; FACCINI, D.; MONTERO, G.; LIETTI, M. Predación de semillas de Amaranthus quitensis H.B.K. en un cultivo de soja: influencia del sistema de siembra. Pesquisa Agropecuária Brasileira, Brasília, v.34, n.3, p.377-384, 1999.

NOONAN, G.R. South American species of the subgenus Anisotarus Chaudoir (genus Notiobia Perty: Carabidae: Coleoptera). Part I: Taxonomy and Natural History. Wisconsin : Milwaukee Public Museum Press, 1981. 85p. (Contribution in Biology and Geology Series).

PETETIN, C.; MOLINARI, E. Reconocimiento de semillas de malezas. Buenos Aires : INTA, 1982. 146p. (Colección Científica del INTA, t.21).

RISCH, S.J.; CARROLL, C.R. Effects of seed predation by a tropical ant on competition among weeds. Ecology, v.67, n.5, p.1319-1327, Oct. 1986.

SAS INSTITUTE. SAS user's guide: Statistics. Cary, NC, 1982. 584p.

SCOPEL, A.L.; BALLARE, C.L.; GHERSA, C.M. Role of seed reproduction in the population ecology of Sorghum halepense in maize crops. Journal of Applied Ecology, v.25, p.951-962, 1988.

SIMPSON, R.L.; LECK, M.A.; PARKER, V.T. Seed banks: general concepts and methodological issues. In:

Pesq. agropec. bras., Brasília, v.35, n.2, p.331-340, fev. 2000 
LECK,M.A;PARKER,V.T.; SIMPSON, R.L. (Eds.) Ecology of soil seed banks. New York : Academic, 1989. Ch.1, p.3-8.

SUDD J.H.; FRANKS, N.R. The behavioural ecology of ants. London : Blackie \& Son Limited, 1987.203p.

TEVIS, L. Interrelations between the harvester ant Veromessor pergandei (Mayr), and some desert ephemerals. Ecology, v.39, n.4, p.695-704, Oct. 1958.

VAN ESSO, M.L.; GHERSA, C.M. Dynamics of Sorghum halepense seeds in the soil of an uncultivated field.
Canadian Journal of Botany, v.67, p.940-944, 1989.

WEAVER, S.E.; McWILLIAMS, E.L. The biology of Canadian weeds. 44. Amaranthus retroflexus L., A. powellii S. Wats. and $A$. hybridus L. Canadian Journal of Plant Science, v.60, p.1215-1234, Oct. 1980.

WHITFORD, W.G. Foraging in seed-harvester ants Pogonomyrmex spp. Ecology, v.59, n.1, p.185-189, Winter 1978. 